\title{
Design of an active dynamic balancing head for rotor and its balancing error analysis
}

\author{
Zhang SHIHAI* and Zhang ZIMIAO** \\ * College of Mechanical Engineering, Tianjin University of Technology and Education, \\ East Liulin, Hexi District, Tianjin 300222, CHINA \\ E-mail: zshky77@163.com \\ ** Tianjin Key Laboratory of High Speed Cutting and Precision Machining, \\ East Liulin, Hexi District, Tianjin 300222, CHINA
}

\section{Recei ved 28 September 2014}

\begin{abstract}
The unbalancing vibration monitoring and control of rotating machinery is an important engineering problem. In order to correct the unbalance of rotor system online, an active dynamic balancing head is designed based on the ratchet-pawl mechanis $m$ and pneumatic technology. The inner mass distribution state of balancing head can be changed to correct the unbalance when the rotor system is working in unbalancing condition. The mechanical principle and pneu matic control system of the balancing head are introduced in the paper. Based on a double-face online dynamic balancing experiment system, the balancing effect of the balancing head has been proved by many experiments. In order to improve the dynamic balancing accuracy of the balancing head in the practical application, the possible influencing factors of dynamic balancing accuracy are analy zed in the paper.
\end{abstract}

Key words :Rotor, Active control, On line dynamic balance, Balancing head, Error analysis

\section{Introduction}

When the rotor is working at high speed, the huge centrifugal force may be caused by the minor unbalance, which will arouse the rotor system to vibrate abnormally. The wear condition between the supporting parts of the rotor system may be exacerbated by unbalancing vibration, so the work efficiency and life of the rotating machinery will be decreased. Therefore the dynamic balance is one of the key problems which must be solved during the designing and manufacturing course of rotating machinery.

The balancing head is the actuator of dynamic balancing system, and its function is to provide the suitable correction for the unbalancing rotor system. At present, the balancing head can be classified to passive and active models based on its work principle. The principle of passive balancing head can be expressed that the unbalance of rotor system is compensated by the centrifugal force of inner weight. It has been proved that the balancing accuracy of the passive balancing head is low in practice. The principle of the active balancing head can be expressed that the unbalance of the rotor system is compensated by outer inputting energy. The active balancing head can be classified to two types based on its work principle. One type is that the outer force is directly exerted on rotor system, in which the electromagnetic force is the common application form. Because the outer force must be exerted continuously during the rotor working time, the continuous energy consumption is inevitable, so this kind balancing head does not apply to the long time running rotor. The other type is that the unbalance of rotor system can be compensated by changing the inner mass distribution of balancing head. This balancing way has the advantage of good adaptability, less energy consumption, no pollution, no effect on the strength of rotor system etc. Therefore it is the hot point of research and application in the active dynamic balance field. The main types and research status of active balancing head is summarized as follows.

The liquid jetting type balancing head. Martin proposed the concept of liquid jetting type balancing head, of which 
the unbalance of rotor can be corrected by changing the mass distribution status of balancing head, and the suitable volume of water is jetted into the corresponding chamber to change the mass distribution status of balancing head during the balancing period (Martin, 1980). Ma et al put forward a new algorith $\mathrm{m}$ for the hydraulic auto balance of online grinding wheels. It comprises the balancing method of cavity by cavity comparison (Ma and $\mathrm{Ji}, 1997$ ). $\mathrm{He}$ developed a new auto-balancing head to solve the problem that the liquid can be projected into the head but can't be released (He, 2001). Gao et al studied the balancing head with controllable liquid impact. The impact is produced by continuous spray and its feasibility has been proved by simulation analysis (Gao and Zhang, 2006). Zhang et al designed a Hydro-jet type balancing head for high speed machine tool spindle. The aim of laminar motion of balancing liquid is realized according to the calculation of the liquid flow rate and the liquid Reynolds number, and the relationship between the balancing capacity and liquid mass is analyzed (Zhang, et al., 2013).

The liquid jetting type of balancing head has the advantage of simplified construction, low cost and easy adjustment etc. But the defects of the balancing head include that the leakage, spattering or volatile of liquid may impact on the balancing precision and environ ment, the balancing operation has to be stopped when the chamber is full of liquid, the liquid will flow out from the chamber outlet when machine tool is stopped and the balancing accuracy is lost.

The motor driving type of balancing head. Vande studied firstly the balancing head with mass blocks, which can be driven by motors and move along the fixed paths (Vande, 1964). Li et al designed an electric balancing head, which is driven by stepping motor and designed to have a ring-like structure with empty centre, and can be adjusted with rolling-car weights and operated in infrared ray remote control manner ( $\mathrm{Li}$, et al., 1998). Ou et al introduced an advanced intelligent system about automatic balance head. The control signal of the motors can be sent out by single-chip microco mputer to adjust the correcting mass of balance head (Ou, et al., 2000).

The motor driv ing type of balancing head has the advantages of high balancing accuracy and easy adjustment etc. But the additional motors are necessary to drive the mass blocks, so the structure and control system of the balancing head is comple $x$ and the cost is high.

The electromagnetic driving type of balancing head. Zeng and Wang designed a new balancing head based on motor principle. The correcting vector of the balancing head can be composed by two balancing discs, and the radial position of balancing discs can be adjusted when the stator coil is plugged in direct current (Zeng and Wang, 1998 ). Dyer and Ni designed an electro magnetic driving type balancing head. The balancing head consists of two unbalancing rings and can be viewed as two heavy spots. When the balancing head is not activated, these two rings are held in place by a permanent magnetic force. The two heavy spots can rotate with the spindle and also be controlled to rotate with respect to the spindle. The combination of these two heavy spots is equivalent to a single heavy spot whose magnitude and position can be changed(Dyer and $\mathrm{Ni}, 2001$ ). Moon and Hredzak respectively developed an electromagnetic driving type of balancing head based on the similar principle (Moon, et al., 2006, Hredzak and Guo, 2006). Fan et al proposed the kinematic precept of mass block in automatic balancer following the principle of online automatic dynamic balancing. By combination of electro magnet and permanent magnet, the magnetic circuit of the balancer with ring coil and rare earth permanent magnet is designed (Fan, et al., 2013).

The electromagnetic driving type of balancing head has the advantages of high balancing accuracy, stable and reliable system etc. But the structure and control system of the balancing head is complex, and the electromagnetic control signal has some interference for rotor control system.

In present, there are some other solutions of online dynamic balance for rotor studied by many relevant scholars. For example, Japanese scholars Nakamoto et al have taken magnetofluid as the balancing correcting mass and designed a new online balancing device. The distributional condition of internal magnetofluid can be changed by controlling the distributional condition of external magnetic field of the balancing head (Nakamoto, et al., 2008). Chinese scholars Wu et al have designed a fractional type of balancing head. The balancing disc with unbalance mass can be driven by the fractional force of rolling bearing during the balance correcting course, and then the rotating speed differences between the balancing discs and rotor spindle are formed and the correcting mass can be adjusted to suitable radial position (Wu, et al., 2008).

Based on the above analysis and considered of the structure characteristics of rotating machinery, a pure mechanical dynamic balancing head has been designed for high-speed rotor system in the paper.

\section{The online dynamic balancing principle based on influence coefficient method}


The influence coefficient method is the common method applied in rotor online dynamic balance. The control process can be divided into two stages of offline experiment and online control, and the basic principle of the method is described as follows.

The purpose of the offline experiment is to test the influence coefficients and save these coefficients to the control system. For explanatory purposes, the number of monitoring points is assumed as $n$, and the number of correcting faces is assumed as $\mathrm{m}$. The offline experimental steps are described as follows.

Step 1, test the initial unbalancing vibration signals of the monitoring points. When the composite correct ion of all the correcting faces are adjusted to 0 (the adjustment principle of composite correction is expressed in part 3 ), the initial vibration signals of points 1 to $\mathrm{n}$ are measured at the balance revolving speed and the unbalancing vibration signals are separated. The initial unbalancing vibration signals of points 1 to $\mathrm{n}$ are respectively assumed as $\overrightarrow{V_{1,0}}=V_{1,0} \angle \varphi_{1,0}, \overrightarrow{V_{2,0}}=V_{2,0} \angle \varphi_{2,0}, \ldots, \overrightarrow{V_{n, 0}}=V_{n, 0} \angle \varphi_{n, 0}$, and the vector matrix of the initial unbalancing vibration is expressed as Eq. (1).

$$
V_{0}=\left[\begin{array}{llll}
\overrightarrow{V_{1,0}} & \overrightarrow{V_{2,0}} & \cdots & \overrightarrow{V_{n, 0}}
\end{array}\right]^{T}
$$

Step 2, test weight in the face $i$. Assume the composite correction imposed in face $\mathrm{i}(\mathrm{i}=1,2, \ldots, \mathrm{m})$ as $\overrightarrow{\mathrm{W}}_{i}$, and the composite correction in other faces are adjusted to 0 , the vibration signals of points 1 to $\mathrm{n}$ are measured at the balance revolving speed and the unbalancing vibration signals are separated. The separated unbalancing vibration signals of points 1 to $\mathrm{n}$ can be taken as the compositive response of the initial unbalance and correction, and the vibration vectors are respectively assumed as $\overrightarrow{V_{1, i}}=V_{1, i} \angle \varphi_{1, i}, \overrightarrow{V_{2, i}}=V_{2, i} \angle \varphi_{2, i}, \ldots, \overrightarrow{V_{n, i}}=V_{n, i} \angle \varphi_{n, i}$.

Based on the linear superposition principle, the influence coefficient vectors of the correcting face i respect to points 1 to $\mathrm{n}$ can be respectively calculated through the Eq. (2).

$$
\overrightarrow{K_{i 1}}=\frac{\overrightarrow{V_{1, i}}-\overrightarrow{V_{1,0}}}{\vec{W}_{i}}, \quad \overrightarrow{K_{i 2}}=\frac{\overrightarrow{V_{2, i}}-\overrightarrow{V_{2,0}}}{\vec{W}_{i}}, \ldots, \quad \overrightarrow{K_{i n}}=\frac{\overrightarrow{V_{n, i}}-\overrightarrow{V_{n, 0}}}{\vec{W}_{i}}
$$

In the Eq. (2), $\overrightarrow{K_{i j}}$ is the influence coefficient vectors of the correcting face i respect to point $\mathrm{j}(\mathrm{j}=1,2, \ldots, \mathrm{n})$.

Based on the same method of step 2, the influence coefficient matrix can be built by $\mathrm{m}$ times test weight experiments, and the matrix is expressed as Eq. (3).

$$
K=\left[\begin{array}{cccc}
\overrightarrow{K_{11}} & \overrightarrow{K_{21}} & \cdots & \overrightarrow{K_{m 1}} \\
\overrightarrow{K_{12}} & \overrightarrow{K_{22}} & \cdots & \overrightarrow{K_{m 2}} \\
\vdots & \vdots & \vdots & \vdots \\
\overrightarrow{K_{1 n}} & \overrightarrow{K_{2 n}} & \cdots & \overrightarrow{K_{m n}}
\end{array}\right]
$$

Step 3, save parameters. The parameters of the influence coefficient matrix need to be saved and used to calculate the control parameters online. In order to improve the precision of those parameters, many offline experiments are performed and their means are taken as the ultimate parameters.

The purpose of the online control is to minimize the unbalancing vibration amplitude of the points 1 to $\mathrm{n}$, and the online control steps are as follows:

Step 1, monitor and analyze the unbalancing vibration condition of rotor system. The vibration monitoring system online acquires the vibration signals of the points 1 to $\mathrm{n}$ at regular intervals, and then the control system separates the unbalancing vibration components from the measuring signals in real time. The balancing correcting function will be carried out when the unbalancing vibration a mplitudes of the monitoring points have exceeded the allowable range. For explanatory purposes, the unbalancing vibration vectors in Eq. (1) are taken as the balancing target.

Step 2, calculate the correction of the correcting faces. The correction matrix of faces 1 to $\mathrm{m}$ is assumed as Eq. (4).

$$
Q=\left[\begin{array}{llll}
\overrightarrow{Q_{1}} & \overrightarrow{Q_{2}} & \cdots & \overrightarrow{Q_{m}}
\end{array}\right]^{T}
$$

In order to eliminate the unbalancing vibration vector $V_{0}$, the Eq. (5) can be built based on the influence coefficient method.

$$
K Q+V_{0}=0
$$

The unique solution of $\mathrm{Q}$ can be solved from Eq. (5) only if the $\mathrm{Q}$ is a square nonsingular matrix, and the 
unbalancing vibration amplitude of monitoring points can be corrected to zero in theory. Usually, the number of correcting faces is less than the number of monitoring points, and the solution of Eq. (5) is not unique. The least square method is applied usually to calculate the value of $\mathrm{Q}$ so as to minimize residual unbalancing vibrations of monitoring points.

The $\mathrm{Q}$ is the essential data for online control. In theory, one correcting face can meet the demands of online dynamic balance for single plane rotor system. Two correcting faces can meet the demands of online dynamic balance for rigid rotor system. Two or more correcting faces are necessary to meet the demands of online dynamic balance for flexible rotor system. How to adjust the correction of correcting face to meet the control demand is the key problem of the online dynamic balance technology.

\section{The online dynamic balancing head}

The online dynamic balancing system for rotor is shown as Fig. 1. For illustration purposes, the product of unbalancing mass and its rotating radius is defined as the fixed unbalance of balancing disc, and the radial position of the unbalancing mass relative to reference point is defined as the phase of balancing disc. In Fig. 1, the correction in a correcting face is composited by two balancing discs, and the vector synthesis principle is shown as Fig. 3.

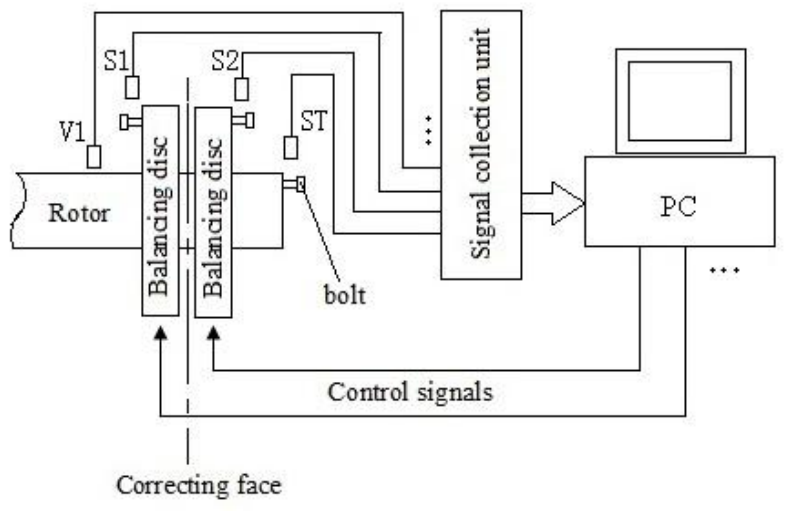

Fig. 1 The online dynamic balance system for rotor

In Fig. 1, the eddy current sensor V1 is used to monitor the vibration signal of point 1 . The photoelectric sensor ST is used to monitor the reference signal of rotor. The photoelectric sensors S1 and S2 are respectively used to monitor the reference signal of the balancing discs 1 and 2. According to the actual structure of balancing disc (in Fig.3), the unbalancing hole is thread structure, and a bolt is fixed in balancing disc through the thread hole. During the condition monitoring period, a positive pulse signal will be outputted from photoelectric sensor when the overhanging part of bolt crosses the dead ahead of photoelectric sensor, and this pulse signal can be taken as the reference of balancing disc. In the same principle, the reference signal of rotor can be gained through a bolt in rotor end. The reference signals of balancing disc and rotor are acquired synchronously, and then the phase of balancing disc can be decided by comparing the reference signals of balancing disc with that of rotor. The monitoring principle of the phase is shown in Fig. 2 .

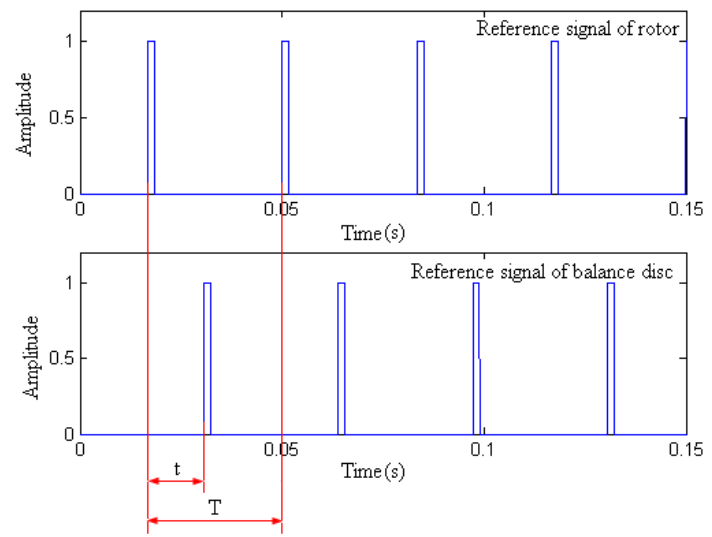

Fig. 2 The monitoring principle of the phase 
Based on Fig.2, the phase of balancing disc can be calculated as the following equation.

$$
\phi=\frac{t}{T} \times 360^{\circ}
$$

Based on this structure, the fixed unbalance of balancing disc is formed by the mass difference between the unbalancing hole and the additional bolt. Meanwhile, the fixed unbalance of the balancing disc can be changed by appending gaskets. In the same principle, the mass difference between thread hole and bolt in rotor end can be taken as a part of unbalance of rotor, and the unbalance of rotor can be adjusted according on experimental require ment.

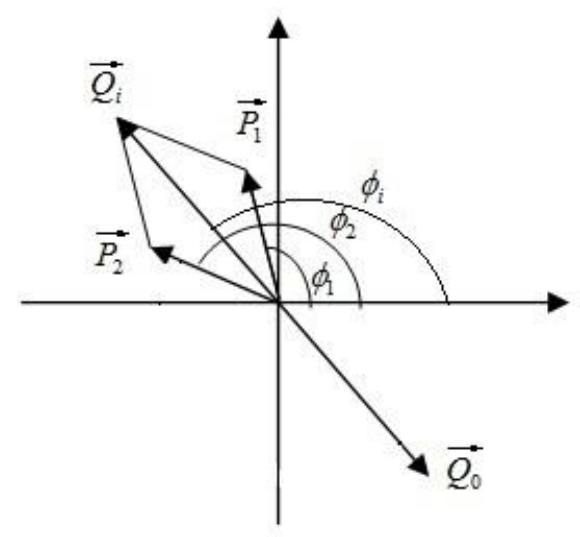

Fig. 3 The vector synthes is principle

In Fig. 3, the $\overrightarrow{Q_{0}}$ is assumed as the equivalent unbalance in correcting face caused by the unbalance of rotor system. The $\overrightarrow{Q_{i}}=P_{i} \angle \phi_{i}$ is assumed as the correction needed to be exerted in the correcting face so as to balance the $\overrightarrow{Q_{0}}$. The $\overrightarrow{P_{1}}=P_{1} \angle \phi_{1}$ and $\overrightarrow{P_{2}}=P_{2} \angle \phi_{2}$ are respectively assumed as the correction caused by balancing disc 1 and 2 in theory. In practice, the fixed unbalance of each balance discs is designed to be equal $\left(\mathrm{P}_{1}=\mathrm{P}_{2}=\mathrm{P}\right)$ so as to easily calculate and control. Based on the vector relation in Fig. 3, the correction of any correcting face can be respectively adjusted by adjusting the phase of corresponding two balancing discs, and the phases $\phi_{1}$ and $\phi_{2}$ of the balancing discs can be calculated based on Eq. (6).

$$
\left\{\begin{array}{l}
\frac{\phi_{1}+\phi_{2}}{2}=\phi_{i} \\
2 P \cos \frac{\phi_{2}-\phi_{1}}{2}=Q_{i}
\end{array}\right.
$$

In equation (6), the $\overrightarrow{Q_{i}}$ can be calculated by influence coefficient method, the $\mathrm{P}$ is the design parameter.

Based on the vector synthes is principle, the correction in a correcting face can be changed by adjusting the phases of corresponding balancing discs. For example, suppose the correction in face 1 is composited by balancing discs 1 and 2 , and the fixed unbalance of two balancing disc is assumed as the same. If the phase difference between balancing discs 1 and 2 is adjusted to $180^{\circ}$, the composited vector in face 1 will be zero. Furthermore, if the phase difference between the balance discs 1 and 2 is adjusted to a value except $180^{\circ}$, the composite correction in face 1 will be a nonzero value.

During the online dynamic balancing period, all the monitoring signals are collected by the signal collection unit and sent to the computer. The unbalancing vibration condition of the spindle is analy zed online by computer according to the monitoring signals. And the correction of correcting faces and the phases of balancing discs are calculated online. Then the control signals are outputted to the driving system and the balancing discs are adjusted to reach their target positions.

Consider of the special requirements of high speed rotor system, a new type online dynamic balancing head has 
been designed based on the principle of ratchet-pawl mechanism. The structure principle of balancing head is shown as Fig. 4.

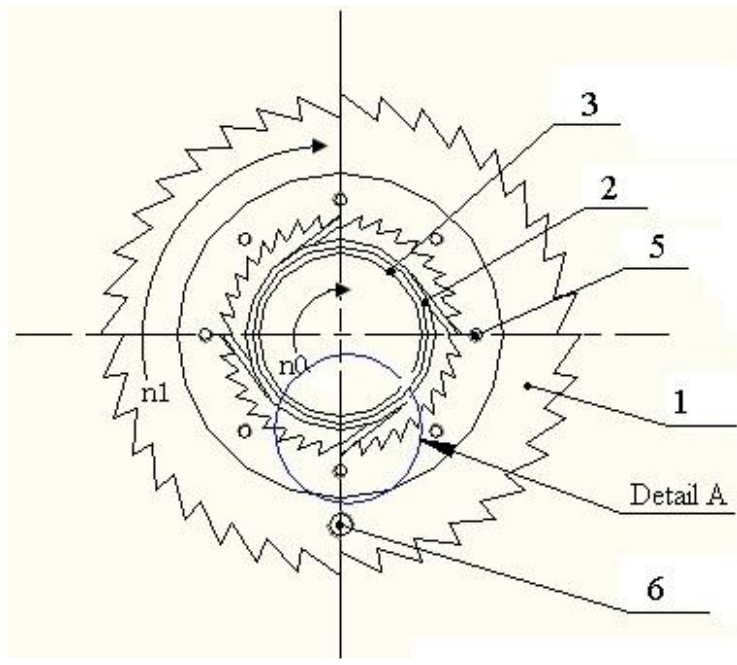

(a)

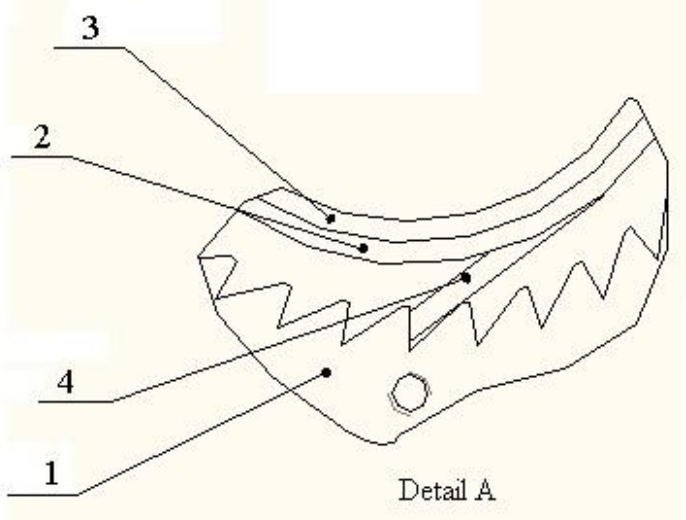

(b)

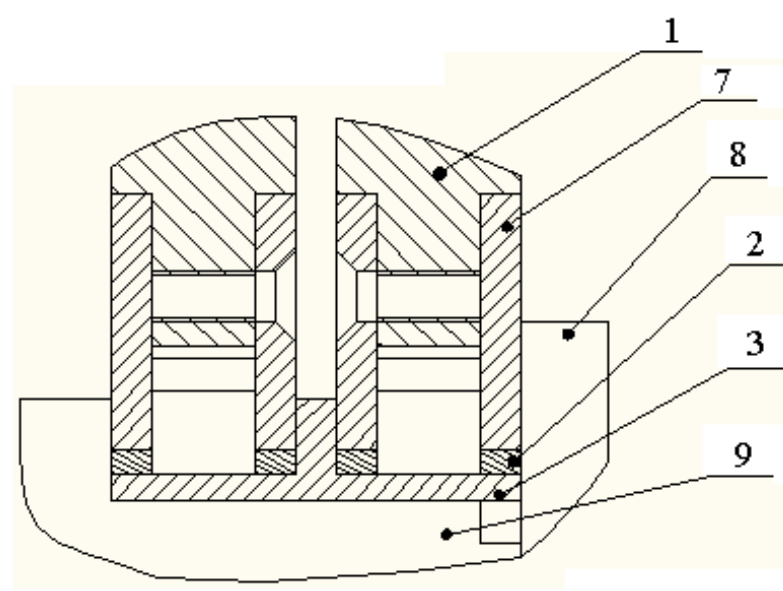

(c)

(d)

Fig. 4 The structure principle of balancing head

1-Balancing disc body, 2-Installing ring, 3-Double shoulder sleeve, 4-Elastic claw, 5-Threaded hole, 6-Unbalancing hole, 7-Side cover, 8-Round nut, 9-Rotating spindle, 10-Balancing head subassembly, 11-Nozzle mechanism

The balancing head is made up of two balancing discs and their installing components. The balancing disc is made up of balancing disc body and two side covers, and each side cover is fixed on the balancing disc body by four sunk screws. The installing components include round nut, double shoulder sleeve, installing ring with four elastic claws etc. The inside and outside circles of balancing disc body are dentate structure, and the unbalancing hole is thread structure. Four elastic claws are uniformly distributed along the outside circle of installing ring.

The assembling relation between the balancing head and rotating spindle is shown as Fig. 4 (a), (b) and (c). Two installing rings are installed respectively on the shoulders of double shoulder sleeve and their installing form is tight fit. The balancing $d$ isc is assembled on the installing ring and the installing form between the inner ring of side covers and the outer ring of installing ring is clearance fit. The ratchet - pawl mechanis $m$ is formed between inside circle of balancing disc and the ends of four elastic claws. The balancing head is assembled on spindle as an integral structure and the installing form between the double shoulder sleeve and spindle is tight fit. The axial position of the balancing disc is located by spindle shoulder, round nut and the flange of double shoulder sleeve, and the suitable tolerance is used to ensure the balancing disc can freely rotate toward one direction relative to spindle. The nozzle mechanism is installed under the balancing disc and their positional relation is shown as Fig. 4 (d).

In Fig.4 (a), the rotating directions of rotor and balancing disc are marked by arrow and their rotating speeds are assumed respectively as $\mathrm{n} 0$ and $\mathrm{n} 1$. When the rotor system is working in balancing condition, the rotating spindle drives the double shoulder sleeves and installing rings to synchronously rotate. Meanwhile the balancing disc is driven to 
synchronously rotate by the elastic claws of installing rings, which results in $n 0=n 1$. On the contrary, when the unbalancing vibration of rotor system exceeds the permitted range, the rotating spindle will be decelerated according to a suitable acceleration, and the high pressure air will be jetted from the nozzles at the same time. The rotating speed of balancing disc can remain unchanged under the combinational force of high pressure air and inertia, which results in $\mathrm{n} 0>\mathrm{n} 1$. When the rotating speed difference $\triangle \mathrm{n}=\mathrm{n} 0$-n1 reaches steady state, the phase of balancing disc can be calculated and adjusted. The high pressure air will be stopped to jet and the rotating spindle will be accelerated to working speed when all the balancing discs are adjusted to their target positions.

The pneumatic control system of the dynamic balancing system is shows as Fig. 5.

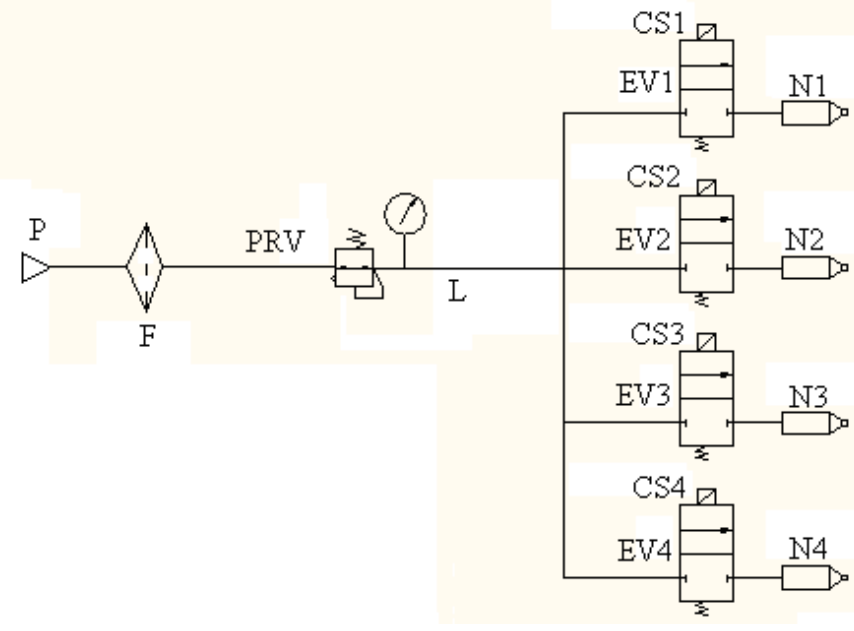

Fig.5 The pneu matic control system

As the Fig. 5 shows, the air pressure of the pipe L can be adjusted through the pressure regulating valve (PRV) according to practical working requirement. When the rotor system runs under the balancing condition, the control signals CS1 to CS4 are 0, and working positions of the two-position two-way valves EV1 to EV4 are shown as Fig. 5. The high pressure air is closed by valves EV1 to EV4. The balancing disc synchronously rotates with rotating spindle. On the contrary, the control signal will be outputted by computer to change the working positions of two-position two-way valves, but the status of control signals CS1 to CS4 depends on whether or not the phase of the corresponding balancing discs needs to be adjusted. For example, if only the phase of the balancing discs 2 and 4 needs to be adjusted, the status of CS1 to CS4 are respectively set by $0,1,0$ and 1 . The high pressure air is respectively jetted from the nozzle 2 (N2) and nozzle 4 (N4), and then the phases of the balancing discs 2 and 4 begin to be adjusted. At the same time, the position sensors monitor the phase of the balancing discs, and send the position signal to the control system in time, then the computer will analy ze whether or not the balance has reached the target position. At some moment, suppose the balancing disc 2 has reached the target position before the balance $\mathrm{disc} 4$, the computer will output the control signal and set the CS2 $=0$, the external air force of the balan cing disc 2 is disappeared, the balancing disc 2 is fixed at the current phase and its rotating speed returns to be synchronous with spindle. Soon after, the balancing disc 4 is adjusted to reach the target position. The status of EV1 to EV4 will return as shown in Fig. 5 and the dynamic balancing operation is finished.

In order to test the balancing effect of the balancing head, the rotor with double-face online dynamic balancing head and their measurement and control system is manufactured. The picture of the experiment system is shown as Fig. 6. 


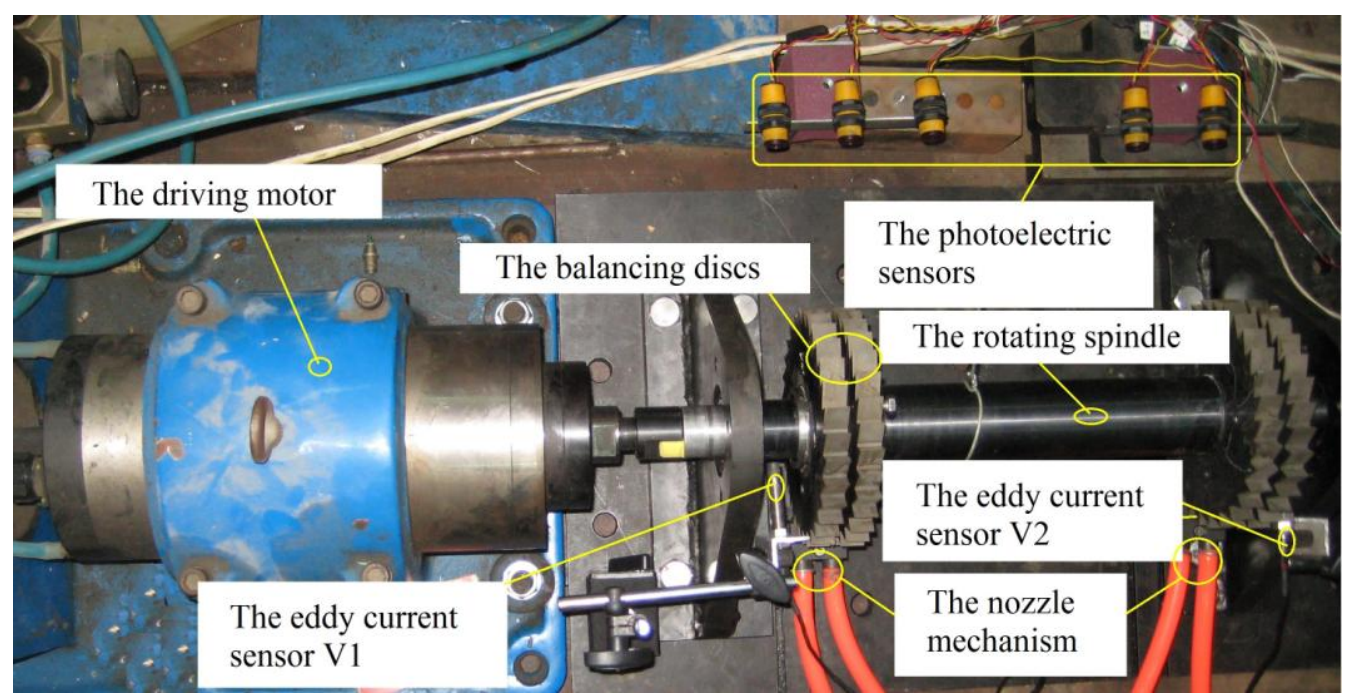

Fig. 6 The experiment system of double-face on line dynamic balance The basic experimental conditions of Fig. 6 are shown in table 1.

Table 1 The experimental conditions

\begin{tabular}{|c|c|c|}
\hline \multirow[t]{2}{*}{ The type of bearings (SKF) } & The front bearing & $7010 \mathrm{CD}$ \\
\hline & The back bearing & $7010 \mathrm{CD}$ \\
\hline \multicolumn{2}{|c|}{ The diameter of rotor $(\mathrm{mm})$} & 75 \\
\hline \multicolumn{2}{|c|}{ The length of rotor (mm) } & 600 \\
\hline \multicolumn{2}{|c|}{ The diameter of balance disk $(\mathrm{mm})$} & 180 \\
\hline \multicolumn{2}{|c|}{ The thickness of balance disk (mm) } & 16 \\
\hline \multicolumn{2}{|c|}{ The fixed unbalance of disk (g.mm) } & 1130 \\
\hline \multicolumn{2}{|c|}{ The balance rotating speed (RPM) } & 1800 \\
\hline \multicolumn{2}{|c|}{ The air pressure $(\mathrm{MPa})$} & 0.8 \\
\hline \multicolumn{2}{|c|}{ The first order natural frequency of rotor in theory $(\mathrm{Hz})$} & 410.28 \\
\hline \multicolumn{2}{|c|}{ The vector of test weight in face 1 and 2} & 1597.59 g. $\mathrm{mm} \angle 45^{\circ}$ \\
\hline
\end{tabular}

Based on the experimental system, a sequence of test weight experiments are performed to test and calculate the influence coefficients. According to the equation (2), the in itial unbalancing vibration of monitoring points, the vector value of test weigh, the unbalancing vibration after respectively test weight in face 1 and 2 need to be acquired. In practical experiment, the mean of many experiments is applied to deduce the signal error. The calculated results of influence coefficients are shown in table 2.

Table 2 The calculation of influence coefficients

\begin{tabular}{c|l|l}
\hline \hline & \multicolumn{1}{|c}{ Point 1 } & \multicolumn{1}{c}{ Point 2} \\
\hline Initial unbalance vibration & $44.61 \mu \mathrm{m} \angle 39^{\circ}$ & $45.48 \mu \mathrm{m} \angle 36.9^{\circ}$ \\
\hline $\begin{array}{c}\text { The unbalance vibration } \\
\text { after test weight in face 1 }\end{array}$ & $239.77 \mu \mathrm{m} \angle 76.4^{\circ}$ & $171.86 \mu \mathrm{m} \angle-80.1^{\circ}$ \\
\hline $\begin{array}{c}\text { The unbalance vibration } \\
\text { after test weight in face 2 }\end{array}$ & $125.22 \mu \mathrm{m} \angle-76.6^{\circ}$ & $233.2 \mu \mathrm{m} \angle 68.3^{\circ}$ \\
\hline Influence coefficients & $\begin{array}{l}\mathrm{K}_{11}=0.1236 \mu \mathrm{m} /(\mathrm{g} \cdot \mathrm{mm}) \angle-136.52^{\mathrm{O}} \\
\mathrm{K}_{21}=0.1224 \mu \mathrm{m} /(\mathrm{g} \cdot \mathrm{mm}) \angle 29.69^{\circ}\end{array}$ & $\begin{array}{l}\mathrm{K}_{12}=0.1213 \mu \mathrm{m} /(\mathrm{g} \cdot \mathrm{mm}) \angle 15.86^{\mathrm{O}} \\
\mathrm{K}_{22}=0.1235 \mu \mathrm{m} /(\mathrm{g} \cdot \mathrm{mm}) \angle-115.17^{\mathrm{O}}\end{array}$ \\
\hline
\end{tabular}

In order to test the balancing effect and stability of the balancing head, a sequence of online dynamic balancing experiments are performed. Three experiment results are shown in table 3 and the time interval of the experiments is one month. 
Table 3 The results of experiments

\begin{tabular}{|c|c|c|c|c|c|c|}
\hline \multicolumn{2}{|c|}{$\begin{array}{l}\text { Experiment times } \\
\text { and monitoring } \\
\text { points }\end{array}$} & $\begin{array}{l}\text { Initial } \\
\text { unbalance } \\
\text { vibration }\end{array}$ & $\begin{array}{l}\text { The theoretical } \\
\text { position of } \\
\text { balance discs }\end{array}$ & $\begin{array}{l}\text { The practical } \\
\text { position of } \\
\text { balance discs }\end{array}$ & $\begin{array}{l}\text { Residual } \\
\text { unbalance } \\
\text { vibration }\end{array}$ & $\begin{array}{l}\text { Balance } \\
\text { quality } \\
\text { grade }\end{array}$ \\
\hline \multirow[t]{2}{*}{1} & Point 1 & $\begin{array}{l}45.61 \mu \mathrm{m} \\
\angle 38.5^{\mathrm{O}}\end{array}$ & \multirow{2}{*}{$\begin{array}{l}\text { Disc 1:-153.45 } \\
\text { Disc 2:-8.67 } \\
\text { Disc 3:-26.39 } \\
\text { Disc 4:-170.23 }\end{array}$} & \multirow{2}{*}{$\begin{array}{l}\text { Disc } 1:-155^{\circ} \\
\text { Disc } 2:-10^{\circ} \\
\text { Disc 3:-25 } \\
\text { Disc 4:-170 }\end{array}$} & $\begin{array}{l}10.52 \mu \mathrm{m} \\
\angle 128.1^{\mathrm{O}}\end{array}$ & G2.5 \\
\hline & Point 2 & $\begin{array}{l}46.46 \mu \mathrm{m} \\
\angle 36.3^{\mathrm{O}}\end{array}$ & & & $\begin{array}{l}10.17 \mu \mathrm{m} \\
\angle-53.7^{\mathrm{O}}\end{array}$ & G2.5 \\
\hline \multirow[t]{2}{*}{2} & Point 1 & $\begin{array}{c}43.79 \mu \mathrm{m} \\
\angle 39.8^{\mathrm{O}}\end{array}$ & \multirow{2}{*}{ 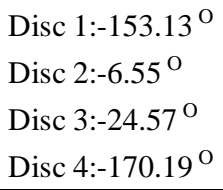 } & \multirow{2}{*}{$\begin{array}{l}\text { Disc } 1:-155^{\mathrm{O}} \\
\text { Disc } 2:-5^{\mathrm{O}} \\
\text { Disc } 3:-25^{\mathrm{O}} \\
\operatorname{Disc} 4:-170^{\mathrm{O}}\end{array}$} & $\begin{array}{l}9.31 \mu \mathrm{m} \\
\angle 125.5^{\mathrm{O}}\end{array}$ & G2.5 \\
\hline & Point 2 & $\begin{array}{l}43.83 \mu \mathrm{m} \\
\angle 37.1^{\mathrm{O}}\end{array}$ & & & $\begin{array}{l}9.92 \mu \mathrm{m} \\
\angle-61.6^{\mathrm{O}}\end{array}$ & G2.5 \\
\hline \multirow[t]{2}{*}{3} & Point 1 & $\begin{array}{l}44.44 \mu \mathrm{m} \\
\angle 38.8^{\mathrm{O}}\end{array}$ & \multirow{2}{*}{ 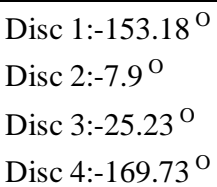 } & \multirow{2}{*}{$\begin{array}{l}\text { Disc } 1:-155^{\mathrm{O}} \\
\text { Disc } 2:-10^{\circ} \\
\text { Disc } 3:-25^{\circ} \\
\text { Disc 4:-170 }\end{array}$} & $\begin{array}{l}8.73 \mu \mathrm{m} \\
\angle 120.2^{\mathrm{O}}\end{array}$ & G2.5 \\
\hline & Point 2 & $\begin{array}{l}46.15 \mu \mathrm{m} \\
\angle 37.4^{\mathrm{O}}\end{array}$ & & & $\begin{array}{l}10.29 \mu \mathrm{m} \\
\angle-57.3^{\mathrm{O}}\end{array}$ & G2.5 \\
\hline
\end{tabular}

The results of experiments indicate that more than 75 percent of the in itial unbalancing vibration can be balanced, and the balance quality grade arrive G2.5 (ISO 1940-1:2003). The balancing effect and stability of the balancing head is ideal.

\section{The balancing error analys is}

The good experiment effect of the balancing head has been gained. In order to improve the balancing precision, the possible errors of the balancing head are analyzed as follows.

\subsection{The signal measurement error}

It is a pre requisite of dynamic balance that the unbalancing vibration signal of rotor is measured accurately. The signal measurement error can be defined as the difference between the measurement value and true value of signal.

Based on the principle of influence coefficient method, the matrix of the influence coefficient must be structured offline by the ways of test weight experiment, signal measurement, signal analy sis and processing, complex operation etc, and then the control signal can be calculated and outputted online based on the influence coefficient matrix. So the adverse influence of balancing precision caused by measurement error is reflected in two aspects. One aspect, when the signal measurement error is produced in the test weight stage, the accuracy of the influence coefficient will be badly affected by the error, and then the accuracy of control signal is badly affected by the inaccurate influence coefficient. The other aspect, when the signal measurement error is produced in the online control stage, the accuracy of control signal is badly affected by the error although the influence coefficient is accurate.

In the practical application, the signal measurement error is unavoidable, which may be caused by the different measurement ways, the precision of sensor and instrument, the nonlinear structure of rotor system, the signal analysis and processing method etc. Therefore the ways of high precision measuring instrument, suitable signal analysis and processing method, many times measure ment and averaging method etc. are applied to decrease the measurement error.

\subsection{The fit clearance error}

Based on the structure principle of the balancing head, the assembly relation among the installing ring, double shoulder sleeve and rotating spindle is tight fit, and the as sembly relation between the balancing disc and installing ring must be clearance fit so as to ensure the phase of balancing disc can be adjusted easily. In the traditional structure, the centre of balancing disc will deviate from the rotary centre of rotating spindle because of the fit clearance, and the additional unbalance will be caused during the rotary period of rotor. The forming principle of the additional unbalance is shown in Fig. 7. 


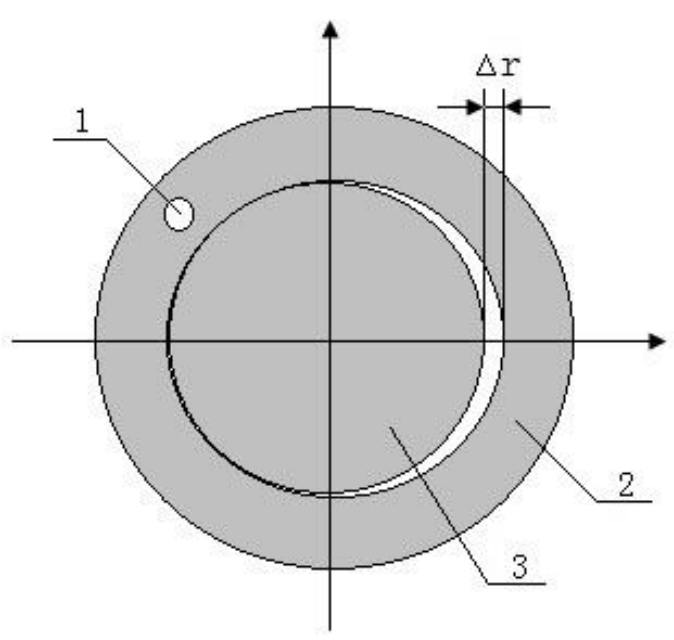

Fig. 7 The forming principle of the additional unbalance

1-unbalancing hole, 2-balancing disc, 3-rotating spindle, $\triangle$ r-offset distance

It can be seen from Fig. 7, the bigger the fit clearance, the better the adjustability of balancing disc, consequently the bigger the offset distance and unbalance. In order to solve the problem, the special structure is designed in the balancing head to compensate the fit clearance and decrease the unbalance. The compensation principle of the fit clearance error is shown in Fig. 8.

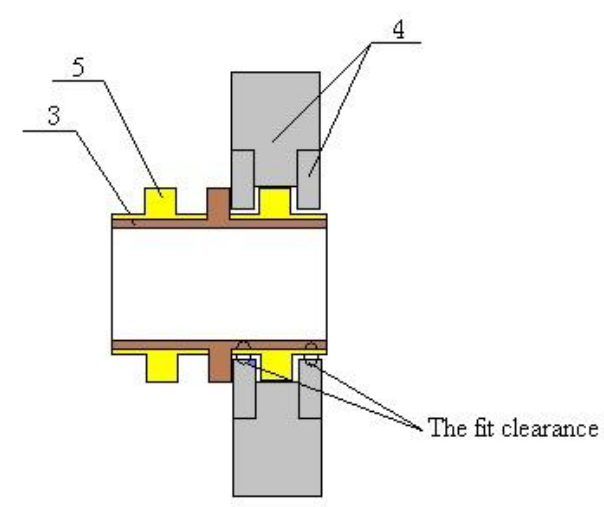

(a)

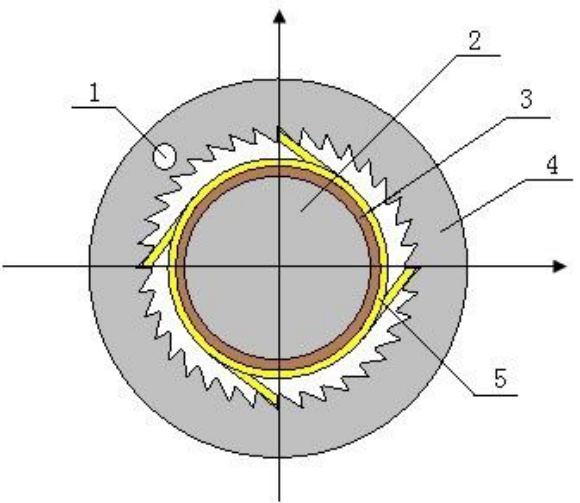

(b)

Fig. 8 The compensation principle of the fit clearance error

(a) The fit relation of the balancing head

(b) The compensation of fit clearance error

1-unbalancing mass, 2-rotating spindle, 3-doube shoulder sleeve, 4-balancing disc, 5-installing ring with elastic claw

The Fig. 8(a) shows that the structure of balancing disc is composed by one balancing disc body and two side covers. The appropriate fit clearance can be ensured by the side covers and installing ring. The Fig. 8(b) shows that four elastic claws are un iformly fitted around the installing ring, and the outward elastic power is continuously exerted on the balancing disc body, so the inhomogeneity of fit clearance can be compensated, and the offset distance and additional unbalance can be reduced.

\subsection{The phase error of the balancing disc}

It can be seen from Fig. 3, the correction in a correcting face is composed by two balancing discs. In order to improve the correcting accuracy, the phase of the balancing discs must be accurately adjusted to the theoretical position. In practice, limited by the number of teeth, the phase of the balancing disc can only be adjusted to reach some limited values. For example, if the number of teeth is 72 , the reachable phases include $0^{\mathrm{O}}, 5^{\mathrm{O}}, \ldots, 355^{\mathrm{O}}$. The nearest number will be selected as the practical phase of balancing disc when the theoretic value locates between any two nu mbers, so the maximum phase error is $2.5^{\mathrm{O}}$ without considering of other factors. In theory, the more the teeth, the s maller the position error, consequently the more difficult to be machined, the higher cost of manufacture, the lower strength of tooth and so on. Furthermore, the phase error of the balancing disc can be influenced by the accuracy of signal 
monitoring and analysis, the accuracy of system control etc. The forming principle of balancing error is shown as Fig. 9 caused by the phase error of the balancing disc.

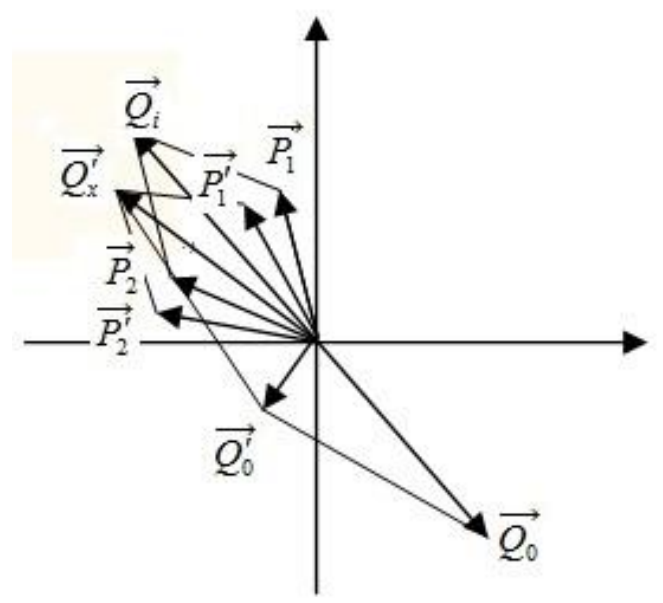

Fig.9 The phase error of balance disc

In Fig. 9, the symbolic meaning of the $\overrightarrow{Q_{0}}, \overrightarrow{Q_{i}}, \overrightarrow{P_{1}}$ and $\overrightarrow{P_{2}}$ is the same as Fig. 3. Because of the phase error, the practical corrections caused by balancing discs 1 and 2 are assumed respectively as $\overrightarrow{P_{1}^{\prime}}$ and $\overrightarrow{P_{2}^{\prime}}$. The practical correction $\overrightarrow{Q_{x}^{\prime}}$ of correcting face is composed by $\overrightarrow{P_{1}^{\prime}}$ and $\overrightarrow{P_{2}^{\prime}}$. It can be seen from Fig. 9, the balancing error $\overrightarrow{Q_{0}^{\prime}}$ is formed by the $\overrightarrow{Q_{0}}$ and $\overrightarrow{Q_{x}^{\prime}}$. In practice, compare with the theoretic phase of balancing disc, the practical phase may be leading or lagging, but the component principle of balancing error is similar.

\subsection{The fixed unbalancing error of balancing disc}

The balancing head is composed by two balancing discs, and the unbalance of the balancing discs is designed to be equal so as to calculate and control easily. However, due to the machining error and material inhomogeneity, the difference of fixed unbalance between the two balancing discs is unavoidable. For easy analysis, the fixed unbalancing error of balancing disc can be defined as the difference between the design value and practical value. The forming principle of balancing error is shown as Fig. 10 caused by the fixed unbalancing error of the balancing disc.

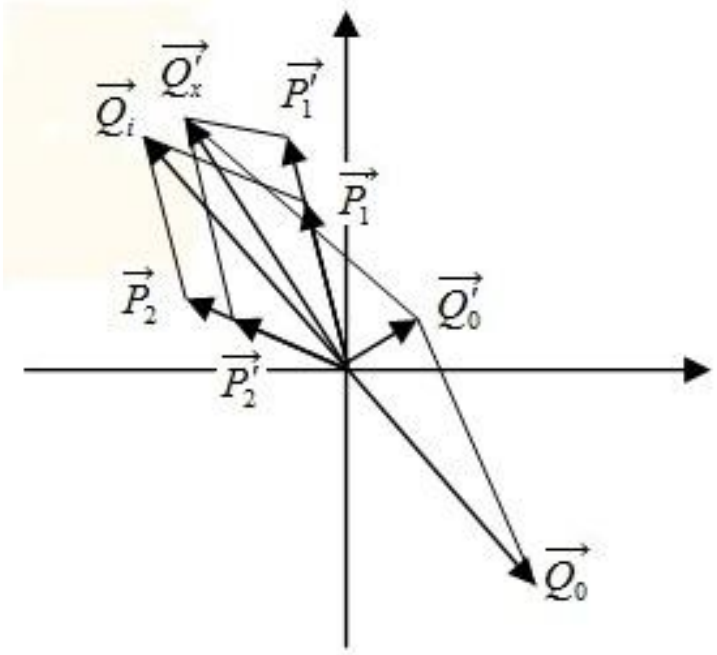

Fig. 10 The fixed unbalancing error of balancing disc 
In Fig. 10, the symbolic meaning of the $\overrightarrow{Q_{0}}, \overrightarrow{Q_{i}}, \overrightarrow{P_{1}}$ and $\overrightarrow{P_{2}}$ is the same as Fig.3. Because of the fixed unbalancing error, the practical corrections caused by balancing discs 1 and 2 are assumed respectively as $\vec{P}_{1}^{\prime}$ and $\overrightarrow{P_{2}^{\prime}}$. The practical correction $\overrightarrow{Q_{x}^{\prime}}$ of correcting face is composed by $\overrightarrow{P_{1}^{\prime}}$ and $\overrightarrow{P_{2}^{\prime}}$. It can be seen from Fig. 10 , the balancing error $\overrightarrow{Q_{0}^{\prime}}$ is formed by the $\overrightarrow{Q_{0}}$ and $\overrightarrow{Q_{x}^{\prime}}$. In practice, the fixed unbalance error may be positive or negative, but the component principle of balancing error is similar. In order to reduce this kind of balancing error, the following methods can be applied.

So me measures are used to improve the machining precision of balancing discs at first. After the balancing disc is machined, the static balance experiments are applied to test the unbalancing error of balancing disc, and then the method of weight removing is used to reduce the unbalancing error.

In practice, it is difficult to completely eliminate the residue unbalancing errors of balancing discs, but the equivalent unbalance of balancing disc can be tested by the method of static balance experiments. In practical dynamic balance operation, the unbalances of balancing discs are taken as not equal, and then the actual unbalances of balancing discs are applied to calculate the position of balancing discs. The balancing error caused by the unbalancing errors of balancing discs can be reduced.

Furthermore, some effective measures are used to reduce the position monitoring and control error, such as improving the installing precision of sensors, improving the rapidity and accuracy of control system, and so on.

\subsection{The installing error of sensors}

The photoelectric sensors are used to monitor the position signals of balancing discs and the reference signal, and the positions of balancing discs benchmarked against the reference signal. So all the photoelectric sensors should be installed in one plane and the centre line of the rotating spindle should lie in the plane. The position adjustment error of the balancing disc will be produced by the installing error of the photoelectric sensors. The balancing error caused by the installing error of photoelectric sensors is similar to the position adjustment error of the balancing disc.

The eddy current sensors are used to measure the vibration signals of monitoring points. All the eddy current sensors should be installed in one plane and their centre line should be vertical and intersecting with the centre line of rotating spindle. Otherwise, the measure error of the vibration signal will be increased by the installing error of the eddy sensors.

\section{Conclusions}

A pure mechanical dynamic balancing head has been designed for high speed rotor in the paper. Compare with similar balancing head, the proposed balancing head has the advantages as follows.

1) The balancing head has the advantages of simple and compact structure, small integral size, especially the axialdimensions is small, which makes the balance head applied in the rotor with compact axial structure. The balancing head is assembled integrally, which makes it easily installed in rotor.

2) The balancing head has the pure mechanical structure and its working stability can't be interfered by outer electromagnetic signal. Meanwhile, there is no electromagnetic signal from balancing head to interfere the working performance of rotor. Therefore the balancing head can be used in the motorized spindle and the rotor with magnetic bearing.

3) The balancing head can be used to control on line the unbalance of high speed rotor by single-face, double-face or multi-face ways, and its balancing effect has been proved high by double-face online dynamic balance experiments.

It is difficult to completely eliminate the unbalance of the rotor system by the online dynamic balance system, but some measures can be utilized to improve the balancing accuracy. The balancing error analysis indicates that the main influencing factors for the balancing accuracy of the balancing head includes the signal measurement error, the fit clearance error, the position adjustment error and the fixed unbalance error of balancing disc, the installing error of 
sensors etc. The fit clearance error can be compensated by the special mechanism of the balancing head, other errors can be decreased by improving machine and installing accuracy, using high precision measurement instrument, utilizing the suitable signal analysis and processing method and so on.

\section{Acknowledge}

This work was supported by the scientific research start-up fund of TUTE (No. KYQD12018).

\section{References}

Dyer, S. W. and Ni, J., Adaptive influence-coefficient control of single-plane active balancing systems for rotating mach inery, Journal of Manufacturing Science and Eng ineering, Vol. 123, No.2 (2001), pp.291-298.

Fan, H. W., Jing, M. Q., Wang, R. C. and Liu, H., Actuating Principle of Online Automatic Balancer with Counter Weight Driven by Magnetic Force, Journal of XI'AN Jiaotong University, Vol.47, No.2 (2013), pp.y1-y6 (in Chinese).

Gao, J. and Zhang, P., Simu lative study of automatic balancing of grinding wheel using a continuously - dripping liquidinjection balancing head, Proceedings of the 16th World Congress on Intelligent Control and Automation (2006)

He, S. Z., Study of liquid release auto-balancing head, Journal of Zhejiang University (Engineering Science), Vol.35, No.4 (2001), pp.418-419 (in Chinese).

Hredzak, B. and Guo, G X, New electromechanical balancing device for active imbalance compensation, Journal of Sound and Vibration, Vol.294, No.2 (2006), pp.737-751.

Li, Y., Liu, Y. G, Lu, Y. P., Xu, G F. and Xia S. B., Electric Balancing Head for High Speed Rotors: Design and Technical Measures, Journal of harbin Institute of Technology, Vol.30, No.3 (1998), pp.1-3 (in Chinese).

Ma, S. X. and Ji, Q. F., Research of a Microcomputer Controlled Hydraulic Autobalance System for on-line Grinding Wheels, Journal of Jilin Institute of Technology, Vol.18, No.4 (1997), pp.4-7 (in Chinese).

Martin, A., Grinding Wheels can Balance Themselves, Machinery and Protection Engr, Vol.136, No.3517 (1980), pp.21-23.

Moon, J. D., Kim, B. S. and Lee, S. H., Development of the active balancing device for high-speed spindle system using influence coefficients, International Journal of Machine Tools and Manufacture, Vo1.46, No.9 (2001), pp.978-987.

Nakamoto, K., Adachi, K. and Shirase, K., Proposal of real-time balancing mechanism using magnetic fluid for mach ine tool spindle, Proceedings of the 41th CIRP Conference on Manufacturing Systems (2008).

Ou, H. T., Zhang, W. Z., Zhang, F. H., Yang, Y. P. and Xu, X. M., Intelligent Wheel Automatic Balancing System Based on Infrared Remote Control of 98 Microcontroller, Measurement \& Control Technology, Vol.19, No.3 (2000), pp.51-53 (in Chinese).

Vande, V. J., Continuous automatic balancing of rotating systems, Journal of Mechanical Engineering Science, Vol.6, No.3 (1964), pp.264-269.

Wu, L. S., Zhang, Y. X., Zhou, D. S. and Zhu, M. L., An automatic dynamic balancing system of fractional type and its control method for high speed spindle, Chinese patent disclosure No.200810102387.6 (2008).

Zeng, S. and Wang, X. X., The electromagnetic balancing regulator and the automatic balancing system, Journal of Sound and Vibration, Vol.209, No.1 (1998), pp.5-13.

Zhang, Y., Mei, X. S., Zou, D. L., Xu, M. X. and Wang, L., Design and Performance Analysis of Hydro jet-typed Balancing Device for High-Speed Machine Tool Spindle, Journal of XI'AN Jiaotong University, Vo1.47, No.3 (2013), pp.y 1-y6 (in Chinese). 